\title{
When it Rains it Pours: Real-time Situational Awareness for Two Weather Emergencies in Connecticut
}

\author{
Kristen Soto*, Jaime Krasnitski, Therese Rabatsky-Ehr and Matthew Cartter \\ Epidemiology, Connecticut Department of Public Health, Hartford, CT, USA
}

\section{Objective}

To characterize the utility of the Connecticut Hospital Emergency Department Syndromic Surveillance (HEDSS) system for real-time situational awareness during two weather-related emergencies.

\section{Introduction}

On August 28, 2011 Tropical Storm Irene made landfall in Connecticut. On October 29, 2011 Connecticut was impacted by Winter Storm Alfred. Both of these storms included high winds and heavy precipitation which resulted in prolonged power outages, disruption of public drinking water systems, property damage, and widespread debris throughout the state. The HEDSS System was utilized to provide real-time situational awareness during the response and recovery phases of both storm events.

\section{Methods}

The HEDSS System receives electronic patient abstract data from 21 of 32 emergency departments on a daily basis. Free-text chief complaint data are characterized into syndrome categories. ED visits for carbon monoxide exposure (CO), gastrointestinal illness (GI), injury, hypothermia, motor vehicle accidents (MVA), and asthma syndromes were monitored throughout the response and recovery periods of both storm. Odds Ratios were calculated using the two weeks preand post-storm as reference dates. $\mathrm{CO}$ visits were further assessed for geographic and demographic trends to target public health messages. The HEDSS system was evaluated to make recommendations for event monitoring during future public health emergencies.

\section{Results}

Following both storms there was a high completeness of daily HEDSS reporting despite extensive power outages (96\% post-tropical storm, 91\% post-winter storm). Increased emergency department utilization for CO (OR: 26.20, 95\% CI: 3.57-192.64) was observed post-tropical storm. Increased emergency department utilization for CO (OR: 14.61, 95\% CI: 7.43-28.72), hypothermia (OR: 17.02, 95\% CI: 3.01-359.30), and asthma (OR: 1.17, 95\% CI: 1.05-1.30) were observed following the winter storm. Regional increases in ED utilization for injuries and MVA were observed following both storm events; no increase in GI was associated with either storm event

During the 2 weeks post-tropical storm 28 cases of CO exposure were reported through HEDSS and 5 cases through laboratory surveillance; during the winter storm 131 cases were reported through
HEDSS and 162 through reportable disease surveillance. Of the 167 cases reported through laboratory surveillance, $111(66 \%)$ were from hospitals that sent data to HEDSS hospitals and 94(56\%) were able to be matched to a specific ED record; of these $22(20 \%)$ were characterized as visiting the ED for CO syndrome, 13(14\%) had symptoms consistent with $\mathrm{CO}$ as their chief complaint, $11(12 \%)$ had smoke/gasoline inhalation exposures as their chief complaint and $2(2 \%)$ had unrelated chief complaints. During the post-storm period the HEDSS systems detected 137 potential CO exposures that were not reported through laboratory surveillance.

\section{Conclusions}

Tropical Storm Irene and Winter Storm Alfred both had significant health impacts, particularly increased ED utilization for CO due to prolonged power outages. The HEDSS system is the only all-hazards surveillance system that was able to provide near-real time information during the storm response phase. In addition to the current CO syndrome definition, where the chief complaint must specifically mention the term $\mathrm{CO}$, a broader definition should also be used in the future to better assess the magnitude of CO-related exposures. The broad definition should include symptomology and related exposures, such as smoke inhalation, to improve case detection. The HEDSS system should continue to be used in conjunction with reportable disease surveillance for situational monitoring during public health emergencies.

\section{Keywords}

emergency department; public health practice; syndromic surveillance; carbon monoxide; weather

\section{Acknowledgments}

Patricia Przysiecki, MPH; Brian Toal, MSPH

\section{References}

1.Witt Associates. Connecticut October 2011 Snowstorm Power Restoration Report. December 21, 2011.http://www.wittassociates.com/assets/860/CTPowerRestorationReport20111201_FINAL_1_.pdf

\section{*Kristen Soto}

E-mail: kristen.soto@ct.gov 